\title{
Delayed Hydrothorax Induced by Suboptimal Positioning of Central Venous Catheter Tip: A Case Report
}

\author{
Bo Young Hwang MD ${ }^{1}$, Hak Jong You $\mathrm{MD}^{1}$, Il Seok Kim MD PhD ${ }^{1 *}$, Min Soo Kim MD
}

${ }^{1}$ Department of Anesthesiology and Pain Medicine, Kangdong Sacred Heart Hospital, Hallym University Medical Center, Seoul, Republic of Korea ${ }^{2}$ Department of Medical Sciences, Hallym University Graduate School, Chuncheon-si, Gangwon-do, Republic of Korea

DOI: $10.36347 /$ sjmcr.2020.v08i01.011

| Received: 08.01.2020 | Accepted: 15.01.2020 | Published: 17.01.2020

*Corresponding author: Il Seok Kim

Abstract

Although advance in technology undergoing central venous access and awareness of consequences have improved, unanticipated complications are still existed. Delayed onset hydrothorax caused by vascular injury from mechanical or chemical irritation of a central venous catheter to the vascular wall may occur if positioning of the catheter is suboptimal. A 55-year-old woman was undergone bypass surgery from the superficial temporal artery to the middle cerebral artery with impression of Moyamoya disease. Before surgery, a triple-lumen central venous catheter was inserted under ultrasound guidance in left subclavian vein for intraoperative hemodynamic managements. The catheter was fixed at a length of $17 \mathrm{~cm}$ from skin punctured site and each port of catheter was functioned optimally. After bypass surgery, she managed with coma therapy in the intensive care unit. Three days after coma therapy, she developed respiratory distress during the weaning from mechanical ventilation. Massive pleural effusion on the right chest, air-bubble with low density fluid collection on the anterior mediastinum, and small amount of pericardial effusion were shown in chest X-ray and computed tomography. Thoracentesis was performed and analysis of pleural fluids showed a transudative pattern with glucose higher than plasma level. With impression of vascular perforation from the catheter to the superior vena cava or innominate vein, sternotomy and pericardiostomy were performed. Although obvious penetrated site of vessel was not found during exploration, abutment with an acute angle and mechanical irritation of catheter tip to mid-portion of the superior vena cava could lead to delayed hydrothorax combined with pericardial effusion. After surgery, she recovered without cardiopulmonary or neurologic sequalae. This case highlights positioning of central venous catheter in left subclavian venous access and assures clinical course of delayed hydrothorax from vascular injury related with catheter.

Keywords: Central Venous Catheters, Hydrothorax, Positioning, Vascular injury.

Copyright @ 2020: This is an open-access article distributed under the terms of the Creative Commons Attribution license which permits unrestricted use, distribution, and reproduction in any medium for non-commercial use (NonCommercial, or CC-BY-NC) provided the original author and source are credited.

\section{INTRODUCTION}

Central venous catheterization has an essential role for hemodynamic managements in perioperative periods. Although advance in technology with ultrasound guidance and awareness of consequences have improved, unanticipated complications are still ongoing.

Hydrothorax caused by suboptimal positioning of a central venous catheter may occur in delayed onset if the catheter tip breaches the vascular wall, resulting in leakage of intravascular fluids into the mediastinum and pleural cavity. Although the incidence of delayed onset vascular injury by a central venous catheter has been reported as low as $0.17-0.4 \%$, delayed recognition and unawareness of clinical course can be fatal to the patients $[1,2]$.
We present a case of delayed onset hydrothorax and pericardial effusion associated with vascular injury following 3 days after catheterization due to suboptimal positioning of central venous catheter undergoing weaning process from coma therapy after bypass surgery in a patient with Moyamoya disease. We also review and discuss the importance of catheter tip positioning of central venous catheterization.

\section{CASE Report}

A 55-year-old woman was admitted to hospital for treatment of left facial palsy, dysarthria and drowsy mentality. On computed tomogram (CT) and magnetic radiographic angiogram, total occlusion in the left distal internal carotid artery, small caliber in the right distal internal carotid artery, non-visualization of both 
proximal middle and anterior cerebral artery, numerous fine basal collaterals, reconstitution of both distal middle cerebral and anterior cerebral artery, and recent infarction on the right middle cerebral territory were seen. By conservative treatment, mentality and dysarthria were improved but left facial palsy was not changed. She was scheduled for bypass surgery from the superficial temporal artery to the middle cerebral artery with impression of Moyamoya disease. She was ASA physical status classification 2 and had a height of $154 \mathrm{~cm}$ and weight of $55 \mathrm{~kg}$. There were no specific findings on physical examination, chest radiography, electrocardiogram, and blood test except new-onset diabetic mellitus before surgery. A peripherally inserted central catheter (PICC) (POWERLINE 5.0 Fr Dual Lumen Catheter, Bard Access System Inc., Salt Lake City, USA) for antibiotic therapy was placed through the right basilic vein due to phlebitis on distal extremities 6 days before surgery.

After induction of anesthesia, a large-bore central venous catheter was inserted to replace blood loss and monitor volume status in perioperative periods. The left subclavian vein was selected because the PICC was existed on the right subclavian vein and the interventional radiographic angiogram was planned on the femoral artery by hybrid operation technique. Subclavian venous catheterization was performed by the Seldinger technique with ultrasound guidance. A triple-lumen central venous catheter (BIOLINE NEXT, EWHA Biomedics Co Ltd, Seoul, Korea) made of latex-free polyurethane with $8.5 \mathrm{Fr}$ in diameter and 20 $\mathrm{cm}$ was inserted. There was neither arterial puncture nor air aspiration during catheterization. After cannulating the subclavian vein, free blood return was observed from all three catheter ports and all ports were easily flushed. The catheter in the vessel lumen was visible on ultrasonography, but the exact location of the catheter tip was not verified. The catheter was fixed at a length of $17 \mathrm{~cm}$ from skin punctured site. The proximal port 5 $\mathrm{cm}$ away from the catheter tip to monitor central venous pressure, the middle port $2.5 \mathrm{~cm}$ away from the catheter tip to administer drugs and the distal port to infuse crystalloid solutions and blood products were functioned optimally. Planned bypass surgery was performed for 9 hours without any event. Total volumes of infused fluid were $4,800 \mathrm{ml}$ of crystalloid, $500 \mathrm{ml}$ of packed RBC and the estimated blood loss was $900 \mathrm{ml}$, urine output was $1,800 \mathrm{ml}$.

After bypass surgery she was extubated and transferred to the intensive care unit (ICU). Postoperative chest X-ray showed no specific abnormality and the tip of central venous catheter placed in the superior vena cava (SVC) (Fig. 1A, 1B).
Arterial blood gas analysis (ABGA) showed $\mathrm{pH} 7.37$, $\mathrm{PCO}_{2} 38 \mathrm{mmHg}, \mathrm{PO}_{2} 130 \mathrm{mmHg}, \mathrm{SaO}_{2} 99 \%$ (FiO2 of $0.5), \mathrm{HCO}_{3}{ }^{-} 22.0 \mathrm{mmol} / \mathrm{L}, \mathrm{BE}-0.2 \mathrm{mmol} / \mathrm{L}, \mathrm{Hb} 11.3$ $\mathrm{g} / \mathrm{dl}$, and Hct $33.6 \%$. During postoperative care, hemodynamics was instable, and hemorrhage transformed around the infarcted area. Coma therapy was started with thiopental sodium, remifentanil, mannitol, nicardipine and inotropic agents following reintubation.

Three days after coma therapy, she was weaned from mechanical ventilation. Six hours after weaning, she complained of dyspnea, developed respiratory distress and $\mathrm{ABGA}$ showed $\mathrm{pH} 7.42, \mathrm{PCO}_{2}$ $35 \mathrm{mmHg}, \mathrm{PO}_{2} 55 \mathrm{mmHg}, \mathrm{SaO}_{2} 89 \%$ (FiO2 of 0.65), $\mathrm{HCO}_{3}{ }^{-} 22.7 \mathrm{mmol} / \mathrm{L}, \mathrm{BE}-1.4 \mathrm{mmol} / \mathrm{L}, \mathrm{Hb} 10.7 \mathrm{~g} / \mathrm{dl}$, and Hct $32 \%$. The chest X-ray revealed massive pleural effusion on the right chest (Fig. 1C). A thoracentesis was performed, and clean fluids were drained from a percutaneous drainage. The CT revealed not only large amount of pleural effusion on the right lung field but also air-bubble with low density fluid collection on the anterior mediastinum and subtle high attenuated pericardial effusion were shown (Fig. 2). Two liters of clear fluids were drained, and analysis of pleural fluids showed a transudative pattern with glucose level of $1254 \mathrm{mg} / \mathrm{dL}$ that was higher than plasma glucose level. In the ICU, vasoactive drugs had been infused with $5 \%$ dextrose in water. These clinical conditions and laboratory findings were caused by leakage of intravascular fluid through the central venous catheter tip out of the wall of SVC or vascular injury by mechanical irritation of catheter to the SVC or innominate vein.

Diagnostic and therapeutic exploration for injured site of vein was performed next day. Median sternotomy and perivascular tissue dissection along the innominate vein and SVC out of pericardial sac were done, but there was no penetrated site along the vein. As the central venous catheter lay inside the SVC still, methylene blue dye solution injected through the central venous catheter for detection of injured site out of the SVC, but there was no extravasation of dye solution. In pericardial sac moderate amount of pericardial effusion was detected. Pericardiostomy was performed and clear fluid was drained, but there was no injured site along the atrium and SVC inside pericardial sac. Complete exploration for pleural and pericardial space following removal of the central venous catheter, thoracotomy was completed without any event. She recovered with postoperative management and was discharged from the hospital 3 weeks after surgery without cardiopulmonary or neurologic sequelae. 

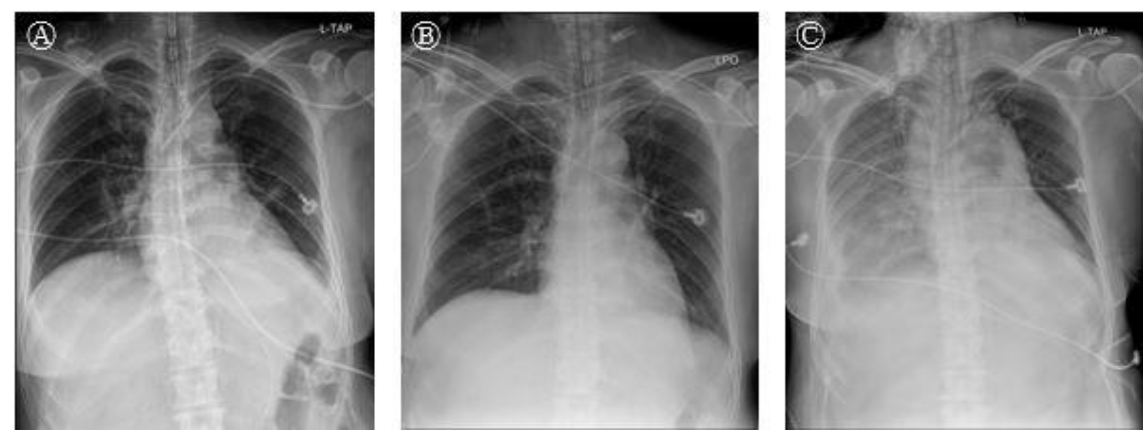

Fig-1: Sequential chest $X$-ray films in the intensive care unit after placement of left subclavian venous catheter. Two central venous catheters were shown at immediate postoperative day. Right-sided catheter was a peripheral inserted central catheter through the basilic vein and leftsided catheter was a large-bore catheter through subclavian vein. Left subclavian venous catheter placed within the superior vena cava, but angle of catheter tip was acute to the vascular wall (A). No specific abnormality was shown at 2 days after coma therapy (B). Three days after coma therapy, the patient developed respiratory distress and massive pleural effusion on the right chest field was shown during weaning from mechanical ventilation $(\mathbf{C})$
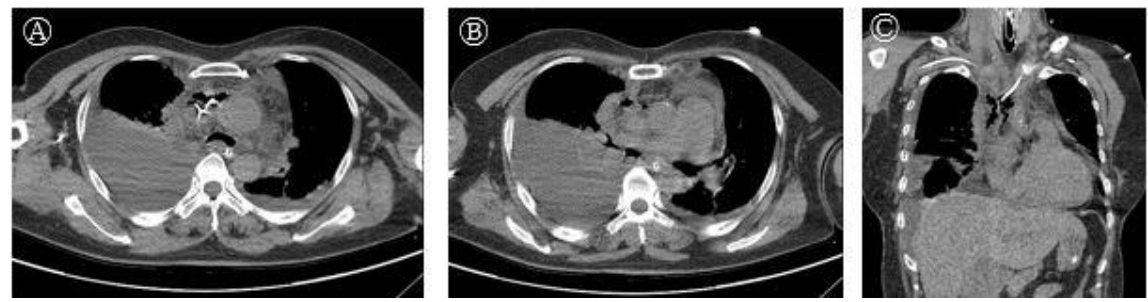

Fig-2: Chest computed tomography images in different levels (A, B) and coronal reconstruction (C). Air-bubble with low density fluid collection suggesting mediastinitis on the anterior mediastinum, subtle high attenuated pericardial effusion and large amount of pleural effusion on the right lung field were shown

\section{DISCUSSION}

We experienced a case of hydrothorax combined with pericardial effusion following 3 days after catheterization due to suboptimal positioning of central venous catheter leading delayed vascular injury in left subclavian venous access.

Vascular injury is mainly categorized as being related to vascular access and catheter maintenance. These are occurred in two separate time sequences [3]. First, direct trauma to the vascular wall during initial catheter inserting procedure by operator error. For example, vascular damage is caused by kinking of a guidewire followed by forceful advancing a dilator. In this early onset injury, vascular perforation is diagnosed within several hours by signs and symptoms of bleeding, hypovolemia or increased intrathoracic pressure. Second, delayed onset vascular injury is caused by mechanical or chemical irritation of catheter tip against the vascular wall in the setting of suboptimal position of catheter tip [4]. Abutment of catheter tip to the vascular wall at an acute angle and cardiac pulsation or patient movement in impinged state of catheter tip against the vascular wall are suggested as mechanical irritation to the vascular wall. Chemical irritation by hyperosmolar solution to the intima leading to phlebitis with edema and necrosis is explained with delayed vascular injury. Delayed onset vascular erosion caused by catheter may occur in several days and manifested as hydromediastinum, mediastinitis, hydrothorax or tamponade. Given that the pericardial sac envelops the middle third of the superior vena cava, vascular perforation below the pericardial sac may induce pericardial effusion or tamponade whereas vascular perforation above the pericardial sac may result in hydromediastinum or hydrothorax.

Some controversy exists with respect of catheter tip positioning in left-sided central venous access [4]. While the catheter tip is positioned in the left innominate vein proximal to the SVC in order to be parallel with the long axis of the vein to prevent vascular perforation, it is risky to thrombosis and dysfunction from relatively small vessel diameter, low blood flow, multiple ports of catheter opening or persistent direct jet of fluid at localized area and feared for infection or dislocation from surface-exposed catheter remnants [5]. In mid position, that is upper and middle SVC, the catheter tip may form a steep angle or abutment to the vascular wall and predispose to impending vascular damage [6,7]. In lower SVC and near cavoatrial junction, parallel arrangement of the catheter tip to the vascular wall reduces the risk of vascular damage and thrombotic complications [3, 4].

Various risk factors for delayed onset vascular erosion are known as left-sided catheter insertion, large bore catheter, rigid catheter material, old age, female gender, administration of cytotoxic agents or hyperosmolar solution for total parenteral nutrition and rapid fluid infusion with high pressure $[1,2]$.

In our case, in spite of these risk factors we inserted relatively large-bore triple lumen central venous catheter in the left subclavian vein for intraoperative hemodynamic managements as other 
sites were hard to selection for being used in different purposes. Although obvious penetrated site of vessel was not found, suboptimal positioning of the catheter tip to the vascular wall led to delayed hydrothorax. Patient movement or cardiac pulsation during weaning process from coma therapy could induce mechanical irritation to the vascular wall. We speculated that vascular erosion site was surrounded by a thrombus which worked as a one-way valve, and therefore a leakage was hardly to detect.

To minimizing preventable complication related catheter maintenance, it is important to establish some strategies. First, prompt removal of unnecessary central venous lines will reduce risks of thrombotic, infectious and mechanical complications. Second, synchronous placement of another central venous catheter within the SVC should be avoided. Third, verifying the catheter tip position will reduce the chance of mechanical and chemical irritation to the vascular wall. We estimated the depth of catheter insertion as 17 cm which derived from Peres' formula based on body height such as (height/10) $+2 \mathrm{~cm}$ for the left subclavian vein [8]. However, this rough estimation is not based on ultrasound guidance but landmark-based catheterization, and moreover it does not consider different skin insertion points depending on the operators. To verify the catheter tip position, several methods such as chest radiography after catheterization, electrocardiography-based method observing the $\mathrm{P}$ wave during catheter placement, and transesophageal echocardiography in real time imaging of catheter tip have been used [9-11]. Unfortunately, as we did not reposition the catheter tip by post-procedural chest radiography nor confirm by echocardiography, delayed hydrothorax occurred due to suboptimal positioning of catheter tip. Vascular injury following abutment of catheter to the SVC wall with an acute angle and mechanical irritation could result in delayed hydrothorax combined with pericardial effusion.

\section{Conclusion}

In conclusion, positioning of central venous catheter tip is very important in left subclavian venous access and the awareness on clinical course of delayed onset vascular injury may reduce such preventable complications.

\section{Footnotes}

Abbreviations: $\mathrm{ABGA}=$ arterial blood gas analysis, $\mathrm{CT}=$ computed tomogram, $\mathrm{ICU}=$ intensive care unit, PICC = peripherally inserted central catheter, $\mathrm{SVC}=$ superior vena cava.

\section{ACKNOWLedGeMENTS}

This research was supported by Basic Science Research Program through the National Research Foundation of Korea (NRF) funded by the Ministry of
Education. (Grant number: NRF2018R1D1A1B07043429)

The institutional review board of Kangdong Sacred Heart Hospital approved this research and waived the requirement for written informed consent because this was a retrospective case report including only 1 patient providing that deal with acceptable treatments and dataset remained de-identified. (IRB No. 2019-04-009)

\section{REFERENCES}

1. Walshe C, Phelan D, Bourke J, Buggy D. Vascular erosion by central venous catheters used for total parenteral nutrition. Intensive Care Med. 2007; 33: $534-537$.

2. Mukau L, Talamini MA, Sitzmann JV. Risk factors for central venous catheter-related vascular erosions. J Parenter Enteral Nutr. 1991; 15: $513-$ 516.

3. Vesely TM. Central venous catheter tip position: a continuing controversy. J Vasc Interv Radiol. 2003; 14: 527-534.

4. Fletcher SJ, Bodenham AR. Safe placement of central venous catheters: where should the tip of the catheter lie? Br J Anaesth. 2000; 85: 188-191.

5. Cadman A, Lawrance JA, Fitzsimmons L, Spencer-Shaw A, Swindell R. To clot or not to clot? That is the question in central venous catheters. Clin Radiol. 2004; 59: 349-355.

6. Gravenstein N, Blackshear RH. In vitro evaluation of relative perforating potential of central venous catheters: comparison of materials, selected models, number of lumens, and angles of incidence to simulated membrane. J Clin Monit. 1991; 7: 1-6.

7. Duntley P, Siever J, Korwes ML, Harpel K, Heffner JE. Vascular erosion by central venous catheters. Clinical features and outcome. Chest. 1992; 101: 1633-1638.

8. Peres PW. Positioning central venous catheters-a prospective survey. Anaesth Intensive Care. 1990; 18: 536-539.

9. Wirsing M, Schummer C, Neumann R, Steenbeck J, Schmidt P, Schummer W. Is traditional reading of the bedside chest radiograph appropriate to detect intraatrial central venous catheter position? Chest. 2008; 134: 527-533.

10. Lee JH, Bahk JH, Ryu HG, Jung CW, Jeon Y. Comparison of the bedside central venous catheter placement techniques: landmark vs electrocardiogram guidance. Br J Anaesth 2009; 102: 662-666.

11. Ender J, Erdoes G, Krohmer E, Olthoff D, Mukherjee C. Transesophageal echocardiography for verification of the position of the electrocardiographically-placed central venous catheter. J Cardiothorac Vasc Anesth._2009; 23: 457-461. 